Check for updates

Cite this: RSC Adv., 2019, 9, 33163

Received 13th July 2019

Accepted 10th October 2019

DOI: $10.1039 / \mathrm{c} 9 \mathrm{ra0} 3365 \mathrm{~d}$

rsc.li/rsc-advances

\title{
Influence of oscillating uniform magnetic field and iron supplementation on quality of freeze-thawed surimi
}

\begin{abstract}
Zhe Wang, ${ }^{\mathrm{ab}}$ Yinying Tan, ${ }^{\mathrm{ab}} \mathrm{Na}$ Yang, (D) *ab Yamei Jin, ${ }^{\mathrm{ab}}$ Han Sun ${ }^{\mathrm{ab}}$ and Xueming $\mathrm{Xu}^{\mathrm{ab}}$
An oscillating uniform magnetic field was applied during the freezing of surimi. Samples were placed at the central zone of a pair of Helmholtz coils (acting as the freezing chamber, guaranteeing a 99\% magnetic field homogeneity) during freeze-thawing. The magnetic field parameters were $4 \mathrm{mT}$ and $50 \mathrm{~Hz}$. After treatments, the physicochemical properties of the samples were immediately investigated. The magnetic field decreased the amount of thawing loss and strengthened the water-holding capacity of the surimi compared to the control. The denaturation temperature, denaturation enthalpy, and protein stability increased as the magnetic field was applied. The addition of ferrous ions (15 mg Fe per $\mathrm{kg}$ surimi, ferrous citrate) enhanced the molecular current under the field due to electromagnetic induction. Morphological observation of the surimi revealed that ferrous ions significantly influenced its structure after freezethawing, yielding the smallest pores among the sample groups. These findings indicate that the quality of freeze-thawed surimi product could be improved by using an oscillating uniform magnetic field combined with iron supplementation.
\end{abstract}

\section{Introduction}

Surimi is a concentrated myofibrillar protein from fish meat that has been washed and dehydrated, and can be used to produce frozen foods with high protein, low fat, and excellent elasticity. Concentrated myofibrillar protein is obtained after washing fish meat to remove most lipids, blood, enzymes, sarcoplasmic proteins, and unnecessary muscle components, which directly contributes to the gelation. ${ }^{1}$ Surimi has many functional properties, including gelling, binding, and emulsifying properties. ${ }^{2,3}$ It can also be used as a raw material for the production of various simulated foods, such as fish balls, simulated shrimp, and crab meat. ${ }^{4}$ Moreover, its functionality and nutritional value can be changed by adding ions, polysaccharides, and proteins..$^{5,6}$ Thus, surimi products are widely welcomed by consumers because of their nutritional value and pleasant taste.

The quality of frozen food depends on moisture distribution and ice crystal size. During slow freezing, the ice nucleusforming rate is slower than the ice crystal growth, resulting in large ice crystal particles in cells and tissues, which degrade the quality of food. ${ }^{7}$ Studies have shown that applying external fields such as microwaves, ${ }^{8}$ ultrasonic waves, ${ }^{9}$ and electric

${ }^{a}$ State Key Laboratory of Food Science and Technology, Jiangnan University, 1800 Lihu Road, Wuxi 214122, PR China. E-mail: yangna@jiangnan.edu.cn; Fax: +86 510 85917100; Tel: +8651085919182

${ }^{b}$ School of Food Science and Technology, Jiangnan University, 1800 Lihu Road, Wuxi 214122, PR China fields ${ }^{10}$ can reduce the size of ice crystal particles during freezing, increase super-cooling temperature, and shorten phase transition time, thus improving the frozen food quality. Although many investigations have been conducted, the effect of magnetic fields on food quality remains controversial. This is because food materials exhibit extremely low magnetic conductivity (relative magnetic permeability, $\mu_{\mathrm{r}} \approx 1$ ) and unknown magnetic field uniformity, which hinders the understanding of the freezing process under a magnetic field.

Zhao et al. (2018) found that the quality of "Fire phoenix" cucumbers could be improved by using an appropriate static magnetic field combined with a cold water shock treatment. ${ }^{11}$ But, Otero et al. (2017a) found that freezing under a weak oscillating magnetic field could not prevent quality loss in frozen crab sticks. ${ }^{12}$ Similarly, freezing under an oscillating magnetic field (maximum value: $418 \mu \mathrm{T}$ ) was not found to significantly affect the freezing characteristics of garlic bulbs. ${ }^{13}$ Moreover, the application of static magnetic fields or oscillating magnetic fields during freezing had no significantly influence on the physicochemical or functional properties of egg components. ${ }^{14,15}$ Based on the existing literature, it is evident that the mechanism by which magnetic field uniformity affects food quality remains unknown.

In recent years, surimi processing has been developed for both marine and freshwater fish to enrich the variety of frozen aquatic foods ${ }^{16}$ In this study, an air-blast freezer equipped with a pair of Helmholtz coils was established. An oscillating magnetic field with high uniformity was then applied to freezing surimi derived from grass carp. Unlike with whole 
pieces of fish meat, adding ferrous ions to surimi is expected to enhance the effect of the magnetic field during freezing due to eddy current. Thus, to explore the effects of ferrous ions and a magnetic field on the physicochemical properties of carp surimi after freeze-thawing, including the freezing parameters, water-holding capacity, thawing loss, differential scanning calorimetry (DSC), and tissue morphology, were analyzed.

\section{Materials and methods}

\subsection{Sample preparation}

Fresh grass carp (Ctenopharyngodon idellus) was purchased from a local supermarket (Wuxi, China). The fish was washed thoroughly with tap water, and the head, scales, skin, spines, and red meat were subsequently removed. Only white meat was picked, it was then carefully washed with ice water $(1: 2 \mathrm{w} / \mathrm{w})$ twice. The meat was cut into fillets, minced and washed with water below $10{ }^{\circ} \mathrm{C}$ at the minced meat-solution ratio of $0.2 \mathrm{~kg}$ $\mathrm{L}^{-1}$ three times (twice with distilled water, then one time with $1.5 \mathrm{~g} \mathrm{~L}^{-1} \mathrm{NaCl}$ aqueous solution, stirred for $5 \mathrm{~min}$ each time and left to stand for $3 \mathrm{~min})$. After dewatering, a traditional cryoprotectant (4\% sorbitol and $0.25 \%$ Na-tripolyphosphate) and ferrous citrate was added into the sample (15 mg Fe per $\mathrm{kg})^{\mathbf{1 7}}$ via a batch-blending process, to ensure that the surimi and additives were thoroughly mixed together. Surimi without the addition of ferrous ions was used as the control. The ferrous content was determined in accordance with the official method outlined by the 2000 AOAC. ${ }^{18}$

\subsection{Experimental system}

An experimental batch air-blast freezer (JD-AC4, INDUC Scientific Co., Ltd., Wuxi, China), which included a magnetic field generator, a fan, a control panel, a freezing chamber, and a refrigeration unit, was used for the freezing. The magnetic field generator consisted of a pair of Helmholtz coils $(80 \mathrm{~cm} \times$ $80 \mathrm{~cm}$ square; 400 turns) and a power supply, and produced a uniform magnetic field at an excitation current of $8 \mathrm{~A}$ with a frequency of $50 \mathrm{~Hz}$. The sample chamber measured $20 \mathrm{~cm} \times$ $20 \mathrm{~cm} \times 20 \mathrm{~cm}$, and the cooling temperature ranged from $-20{ }^{\circ} \mathrm{C}$ to $10{ }^{\circ} \mathrm{C}$. A BLD-1030 Gaussian meter (Bolandun Corporation Ltd., Beijing, China) and a micro-displacement platform were used to measure the field strength and its homogeneity in the chamber. At these settings, the maximum measured magnetic field intensity was $4 \mathrm{mT}$ (AC), and the homogeneity of the magnetic field was $99 \% .^{12,19}$

\subsection{Freeze-thaw procedure}

The experiments were performed using the above-mentioned system. The cooling temperature was fixed at $-20{ }^{\circ} \mathrm{C}$. The surimi was placed in a $25 \mathrm{~mL}$ beaker (35 mm diameter), then covered with plastic film and placed in the freezing chamber. The samples were frozen under the magnetic field until the sample temperature reached $-18{ }^{\circ} \mathrm{C}$ (about $4 \mathrm{~h}$ ). After freezing, the surimi was thawed at $10{ }^{\circ} \mathrm{C}$ under the magnetic field until the sample temperature reached $8{ }^{\circ} \mathrm{C}$ (about $10 \mathrm{~h}$ ). The physicochemical properties of the samples were then immediately analyzed.

\subsection{History of temperature}

During the treatment, type-T (copper/constantan) thermocouple wires (1 mm diameter) connected to a data logger (Eltek Type 1000, Grant Instruments Co., Ltd., Cambridge, UK) were located at the geometric center of the beaker filled with the sample. The sample temperature was then recorded.

\subsection{Thawing loss}

In this study, the term "thawing loss" is used to describe the exudates from the freeze-thawed samples. For each determination, the samples were weighed before freeze-thawing $\left(M_{0}\right)$, and the freeze-thawed samples were blotted dry with absorbent paper and weighed immediately $\left(M_{1}\right)$. Thawing loss was calculated using the following equation:

$$
\text { Thawing loss }(\%)=\frac{\left(M_{0}-M_{1}\right)}{M_{0}} \times 100
$$

where $M_{0}$ and $M_{1}$ are the masses ( $g$ ) of the sample before and after freeze-thawing, respectively.

\subsection{Water-holding capacity}

The water-holding capacity (WHC) of the surimi was measured via centrifugal force to remove the free water. For each determination, approximately $5 \mathrm{~g}$ of the sample was weighed and placed in a centrifuge tube. The tube had a perforated disc, which was covered with 2 pieces of filter paper. The sample was placed on this perforated disc and centrifuged at $2200 \mathrm{~g}$ and $4{ }^{\circ} \mathrm{C}$ for $10 \mathrm{~min}$. After centrifugation, the sample was weighed again. The WHC was calculated using the following equation.

$$
\text { WHC }(\%)=\left(1-\frac{M_{0}-M_{1}}{M_{0} \times m_{\mathrm{tw}}}\right) \times 100
$$

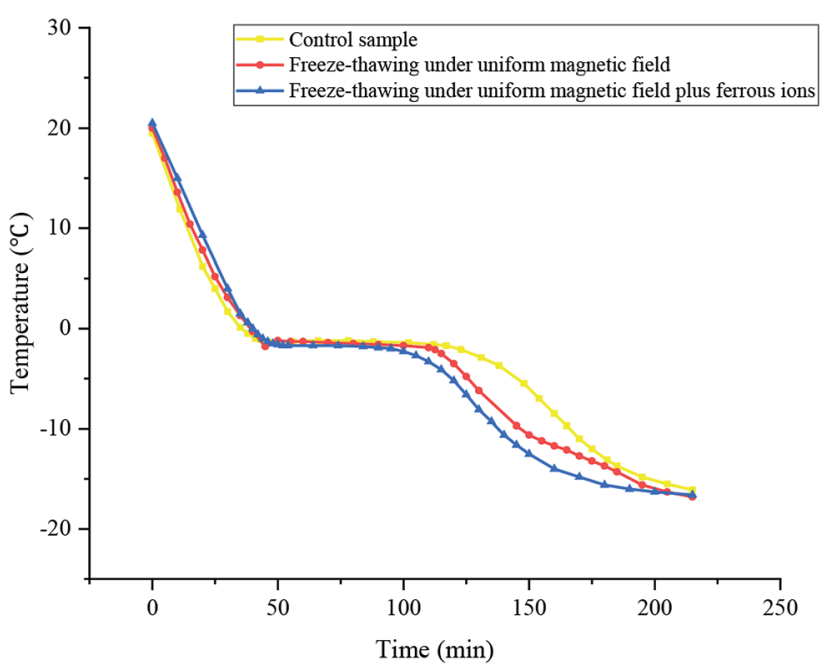

Fig. 1 Influence of uniform magnetic fields on the temperature history of surimi during freezing. 
where $M_{0}$ and $M_{1}$ are the masses ( $\mathrm{g}$ ) of the sample before and after centrifugation, respectively, and $m_{\mathrm{tw}}$ is the mass fraction of the total water present in the sample prior to centrifuging, which was determined by the mass loss after oven-drying at $105{ }^{\circ} \mathrm{C}$ to a constant weight.

\subsection{Texture}

Texture profile analysis (TPA) of the samples was carried out according to Gani \& Benjakul (2018) with a minor modification. ${ }^{20}$ Texture profile analyses (TPA) were performed using a cylindrical measuring probe (P5/S) attached to a TA. A TX2 texture analyzer (TA-XT plus, Stable Micro Systems, Ltd., Surrey, UK) was used at a constant probe speed of $1.0 \mathrm{~mm} \mathrm{~min}^{-1}$ at room temperature $\left(25 \pm 1{ }^{\circ} \mathrm{C}\right)$. The surimi sample was also placed in the $25 \mathrm{~mL}$ beaker. The hardness, springiness, cohesiveness, chewiness, and resilience were determined from the force-time curves.

\subsection{DSC}

The endothermal transitions of the samples were determined using a differential scanning calorimeter (S II Nano Technology Inc., Seiko, Japan). The temperature was calibrated using an indium thermogram. Samples of 8-12 $\mathrm{mg}$ were accurately weighed into standard aluminum pans, sealed and heated from $25{ }^{\circ} \mathrm{C}$ to $90{ }^{\circ} \mathrm{C}$ at a scanning rate of $10^{\circ} \mathrm{C} \mathrm{min}{ }^{-1}$ under a nitrogen atmosphere. An empty pan was set as the reference. The enthalpy values $(\Delta H)$ and peak transition temperature $\left(T_{\max }\right)$ were estimated from the thermogram via Pyris-12 software (PerkinElmer Instruments, USA).

\subsection{Morphology}

Morphological images of the lyophilized surimi were observed using a photon microscope equipped with a digital camera (BT1600, Dandong Bettersize Instruments, Ltd., China).

\subsection{Statistical analysis}

The experiments were performed in triplicate. SPSS software (version 16.0, SPSS, Inc., Chicago, IL) was used to analyze the data. Significant differences in the treatments were evaluated by one-way analysis of variance (ANOVA; 95\% significance level) and Duncan's test. All experimental data are expressed as the mean \pm standard deviation (SD).

\section{Results and discussion}

\subsection{Temperature history and freezing characteristics}

The temperature curves of the samples subjected to different treatments during freezing are presented in Fig. 1. There was no super-cooling phenomenon for the surimi under the magnetic field, which is in accordance with a previous result of the
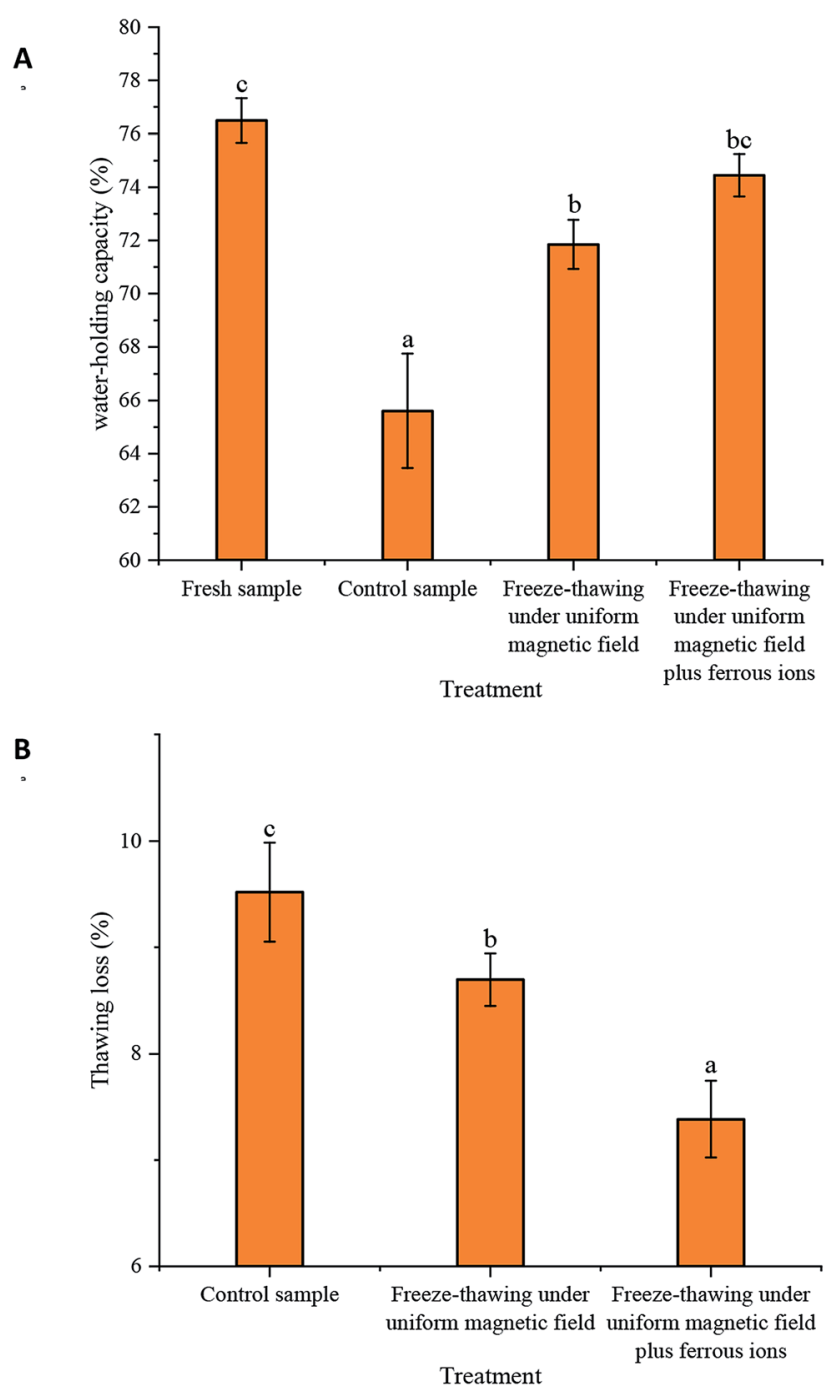

Fig. 2 Water-holding capacity $(p=0.021,<0.05)$ and thawing loss ( $p=$ $0.043,<0.05$ ) of freeze-thawed surimi samples. Different lowercase letters within the same sample indicate significant differences.

Table 1 Freezing characteristics of surimi ${ }^{a}$

\begin{tabular}{lrr}
\hline Conditions & $\begin{array}{l}\text { Freezing point } /{ }^{\circ} \mathrm{C} \\
(p=0.041,<0.05)\end{array}$ & $\begin{array}{l}\text { Phase transformation } \\
\text { time/min }(p=0.028,<0.05)\end{array}$ \\
\hline Control sample & $-1.3 \pm 0.1^{\mathrm{b}}$ & $105 \pm 2^{\mathrm{b}}$ \\
Freeze-thawing under uniform magnetic field & $-1.2 \pm 0.1^{\mathrm{b}}$ & $79 \pm 3^{\mathrm{a}}$ \\
Freeze-thawing under uniform magnetic field plus ferrous ions & $-1.7 \pm 0.1^{\mathrm{a}}$ & $73 \pm 3^{\mathrm{a}}$
\end{tabular}

${ }^{a}$ The different lowercase letters ( $\mathrm{a}$ and b) indicated a significant difference based on the Duncan's test and least significant difference (LSD). 


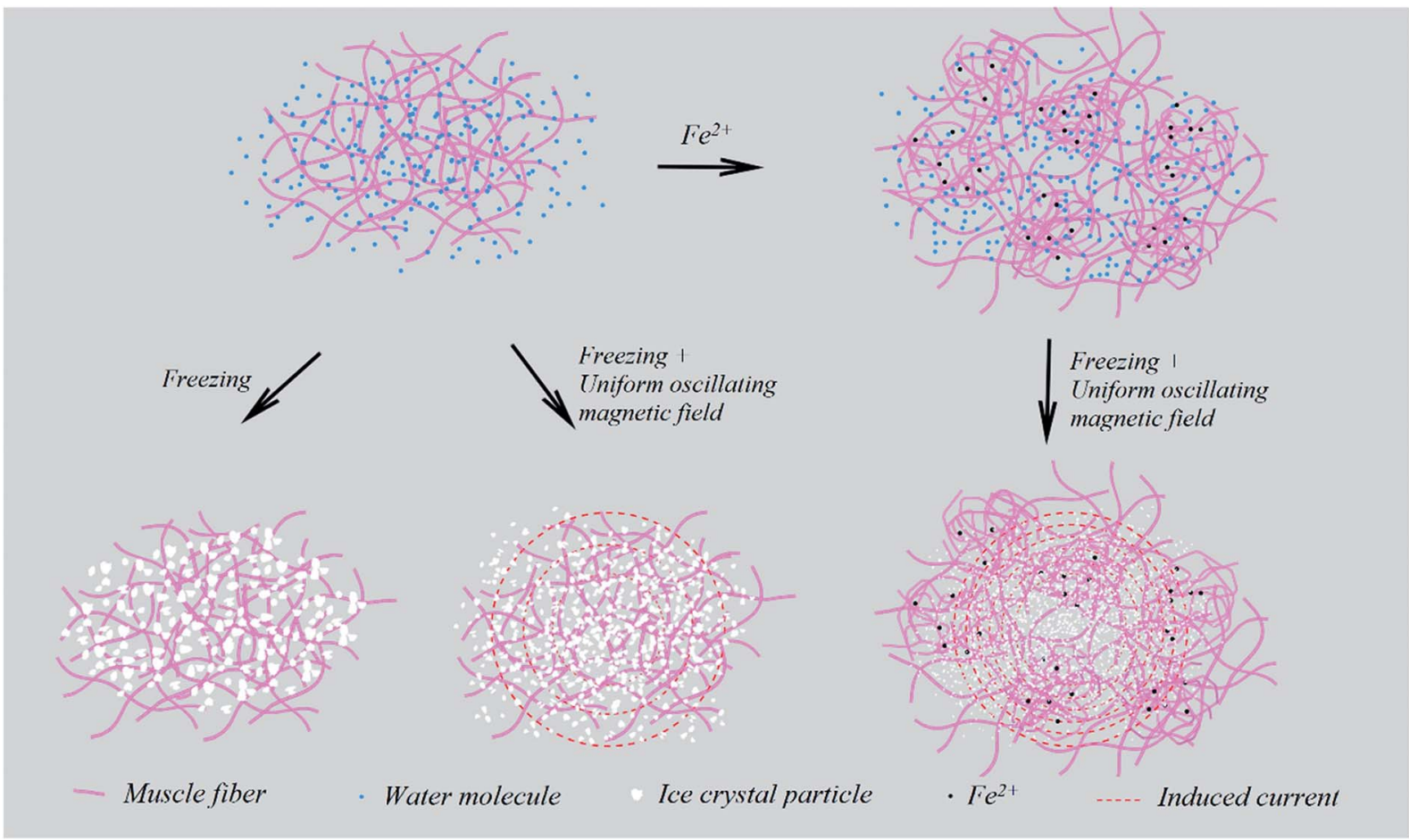

Fig. 3 The effect mechanism of the oscillating uniform magnetic field combined with ferrous ions on surimi during freeze-thawing.

ultrasonic-assisted freezing of surimi. ${ }^{21}$ Table 1 demonstrates that the freezing point and phase transformation time of surimi were reduced under the magnetic field. In the study, the phase transformation time of the sample was defined as the time required for the temperature to decrease by $5{ }^{\circ} \mathrm{C}$ from its freezing point. In comparison with the control, the uniform magnetic field-assisted freezing remarkably shortened the phase transformation time. The lowest time $(73.3 \mathrm{~min})$ in the phase transition of surimi was observed for the sample with ferrous ions, exhibiting a reduction of $43.7 \%$ compared to that of the control (105.3 min). This indicates that the surimi subjected to the temperature zone for maximum ice crystal formation became quickly affected by the non-thermal effect of the magnetic field.

The magnetic field applied to the group of free water molecules induced the damage of hydrogen bonds during the phase transformation stage. In addition, under the uniform magnetic field during freeze-thawing, macro-molecular clusters of water were broken into micro-molecular clusters or even individual molecules, making it difficult to form large ice crystal particles. This accordingly accelerated the phase transformation process. Jin et al. (2015) studied the influence of a static magnetic field (SMF) on $0.9 \% \mathrm{NaCl}$ freezing; their research revealed that the phase transformation time under the repulsive SMF $(1504 \pm 10$ s) was reduced by $32.1 \%$, but it was increased when the attractive SMF was applied. ${ }^{22}$ Furthermore, a study on the influence of SMF on the super-cooling, freezing dynamics of pure water and $0.9 \% \mathrm{NaCl}$ solution was conducted by Otero et al. (2017b). It was found that the SMF (either the attractive SMF or the repulsive SMF) had no remarkable influence on the degree of super-cooling, nucleation temperature, or phase transformation time. ${ }^{23}$

Table 2 Texture parameters of the freeze-thawed surimi and fresh sample ${ }^{a}$

\begin{tabular}{|c|c|c|c|c|c|}
\hline Parameters & $\begin{array}{l}\text { Hardness }(\mathrm{g}) \\
(p=0.04,<0.05)\end{array}$ & $\begin{array}{l}\text { Resilience }(\%) \\
(p=0.03,<0.05)\end{array}$ & $\begin{array}{l}\text { Cohesion } \\
(p=0.01,<0.05)\end{array}$ & $\begin{array}{l}\text { Elasticity } \\
(\%)(p=0.014,<0.05)\end{array}$ & $\begin{array}{l}\text { Chewiness } \\
(p=0.018,<0.05)\end{array}$ \\
\hline Fresh sample & $29.63 \pm 1.92^{\mathrm{a}}$ & $9.70 \pm 0.32^{\mathrm{c}}$ & $0.54 \pm 0.03^{\mathrm{c}}$ & $82.36 \pm 3.51^{\mathrm{c}}$ & $13.25 \pm 1.05^{\mathrm{c}}$ \\
\hline $\begin{array}{l}\text { Freeze-thawing under oscillating } \\
\text { uniform magnetic field }\end{array}$ & $32.40 \pm 0.80^{\mathrm{b}}$ & $8.87 \pm 0.57^{\mathrm{a}, \mathrm{b}}$ & $0.46 \pm 0.01^{\mathrm{b}}$ & $67.48 \pm 4.17^{\mathrm{a}, \mathrm{b}}$ & $10.49 \pm 0.54^{\mathrm{a}, \mathrm{b}}$ \\
\hline Freeze-thawing under oscillating & $35.14 \pm 1.50^{\mathrm{c}}$ & $9.06 \pm 0.56^{\mathrm{b}, \mathrm{c}}$ & $0.46 \pm 0.01^{\mathrm{b}}$ & $72.23 \pm 3.14^{\mathrm{b}}$ & $10.88 \pm 1.25^{\mathrm{b}}$ \\
\hline
\end{tabular}

uniform magnetic field plus

ferrous ions

${ }^{a}$ The different lowercase letters (a-c) indicated a significant difference based on the Duncan's test and least significant difference (LSD). 


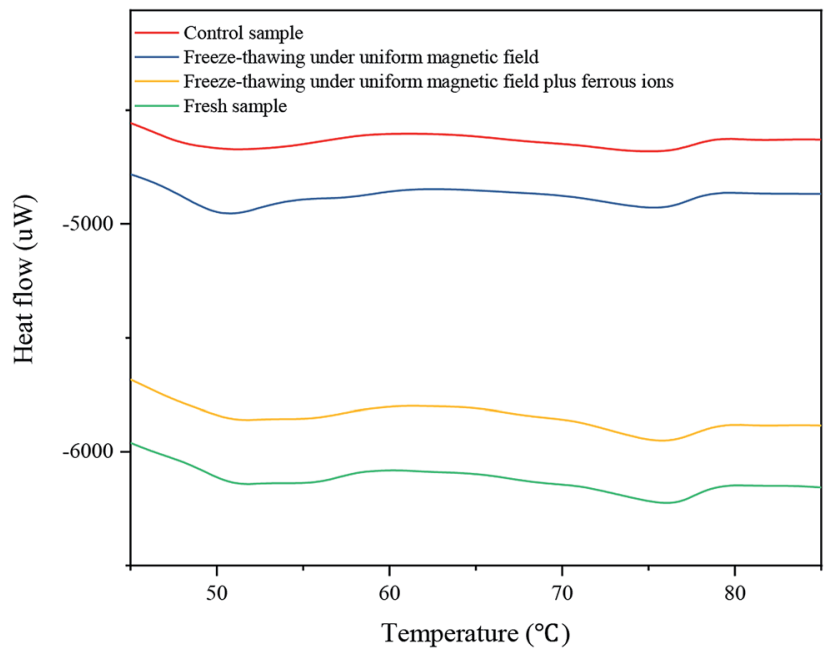

Fig. 4 DSC analysis of the freeze-thawed surimi and fresh sample.

\subsection{Water-holding capacity and thawing loss}

Fig. 2 presents the effect of the uniform magnetic field on waterholding capacity and thawing loss of the samples. The waterholding capacity and thawing loss are important indicators of the quality of surimi. ${ }^{24}$ Increases in thawing loss degrade the quality of food and decrease its weight, ultimately reducing the economic value of the product. ${ }^{25}$ Compared to the control, the magnetic field lower the thawing loss and strengthened the water-holding capacity of the surimi. The higher thawing loss and lower water-holding capacity of the control were attributed to ice crystal growth, which squeezed adjacent muscle fibers and thus decreased the amount of water held by capillary force. Additionally, the damage to muscle fibers causes cellular content leakage and protein degeneration, thus decreasing the water-binding ability of proteins. ${ }^{25,26}$ This trend is dependent on the phase transformation of the sample during freezing. When the transformation time was shortened, smaller ice crystal particles appeared.

Otero et al. (2017a) found that the quality indexes of food were reduced as a magnetic field (magnetic field strength $<3$ $\mathrm{mT}$, oscillating frequency $6-59 \mathrm{~Hz}$ ) was applied during freezing. ${ }^{12}$ Rodríguez et al. (2017) claimed that the application of a weak oscillating magnetic field with different intensities during freezing did not significantly influence the thawing loss of pork loin. ${ }^{27}$ This suggests that the unknown magnetic field uniformity during freezing is the main reason that food quality is sometimes unaffected. Conversely, the application of an oscillating magnetic field with high uniformity to the surimi could have induced small eddy currents in the sample, which affected the freeze-thawing characteristics.

The influence of ferrous ions on the water-holding capacity and thawing loss of surimi was also investigated. Both indexes presented a similar trend after the addition of ferrous ions, resulting in higher water-holding capacity and lower thawing loss. This suggests that the supplementation of iron could further improve these indexes of the sample during freeze-thawing under the magnetic field. Specifically, the water-holding capacity and thawing loss of the sample after the addition of ferrous ions were $76.87 \%$ and $7.39 \%$, respectively, whereas those of the control were $65.61 \%$ and $9.52 \%$, respectively.

It is inferred that two factors contributed to this improvement (Fig. 3). The first is that the added ions enhanced the response of the surimi to an oscillating magnetic field due to eddy current, which broke hydrogen bonds between water molecules and inhibited the aggregation of water molecules during freezing, thus decreasing the size of ice crystals and reducing the damage to muscle fibers. ${ }^{28}$ The other possibility is that the ferrous ions enhanced the cross-linking between proteins, and consequently formed a network structure with a higher water-holding capacity. ${ }^{29}$

\subsection{Texture}

Texture profile analysis (TPA) is a rheological method by which to imitate the mastication process, and these parameters of the samples are exhibited in Table 2. The values of hardness, which represent the force required to compress a sample to attain a given deformation, as well as the resilience, cohesion, elasticity, and chewiness of the fresh surimi were higher than those of the freeze-thawed samples. Moreover, the texture parameters of the surimi were influenced by the application of the magnetic field. The hardness and elasticity of the sample with ferrous ions were $35.14 \mathrm{~g}$ and $72.23 \mathrm{~g}$, respectively, exhibiting increases of $12.4 \%$ and $10.2 \%$ compared to the control. The lower hardness and elasticity levels of the control were due to the further damage of the surimi structure by large ice crystal particles. In

Table 3 Maximum transition temperature $\left(T_{\text {max }}\right)$ and denaturation enthalpy $(\Delta H)$ of myosin (peak I) and actin (peak II) for the surimi samples ${ }^{a}$

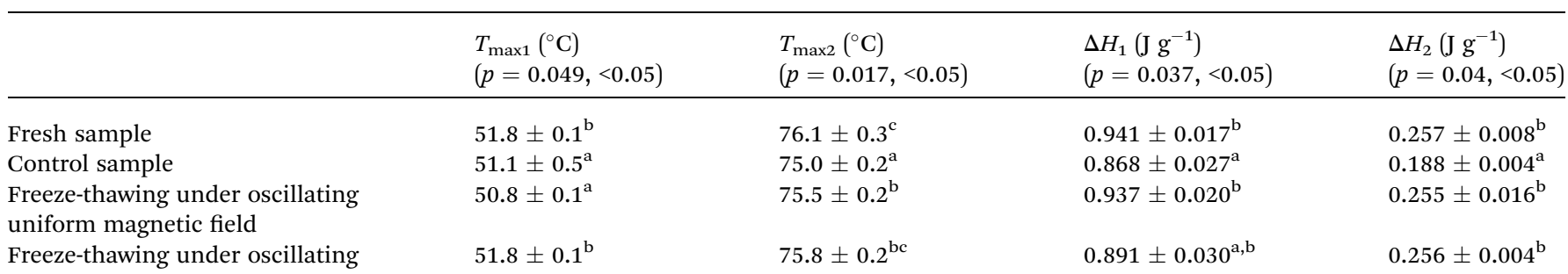

uniform magnetic field plus

ferrous ions

${ }^{a}$ The different lowercase letters (a-c) indicated a significant difference based on the Duncan's test and least significant difference (LSD). 
addition, the surimi sample both under the oscillating uniform magnetic field and with the addition of ferrous ions had higher chewiness, which reflects a better taste, as compared to the control. These parameters were also consistent with the trends of phase transformation time, water-holding capacity, and thawing loss, which indicate that an oscillating uniform magnetic field-assisted freeze-thawing process could improve the quality of surimi.

\subsection{DSC analysis}

Differential scanning calorimetry (DSC) is a thermo-analytical technique that determines the protein denaturation temperature. The peak temperature $\left(T_{\max }\right)$ represents the temperature at which protein denaturation occurs, and reflects the stability of protein structure. The denaturation enthalpy $(\Delta H)$ indicates the amount of energy required for protein denaturation. ${ }^{30}$ Fig. 4 and Table 3 present the thermal results of the surimi samples. Two characteristic peaks appeared at 47.79-55.76 ${ }^{\circ} \mathrm{C}\left(T_{\max 1}\right)$ and 68.23-76.63 ${ }^{\circ} \mathrm{C}\left(T_{\max 2}\right)$, corresponding to the denaturation temperatures of myosin and actin, respectively. ${ }^{25}$ The $T_{\max 1}$ values of the samples exposed to the magnetic field and with the ions were $51.1^{\circ} \mathrm{C}$ and $51.8{ }^{\circ} \mathrm{C}$; in contrast, the $T_{\max 2}$ values were $74.95{ }^{\circ} \mathrm{C}$ and $75.8{ }^{\circ} \mathrm{C}$, respectively. This demonstrates that the freeze-thawed surimi with ferrous ions had the highest $T_{\max }$ and $T_{\max 2}$ values, and therefore the highest thermal stability, under the magnetic field. The $\Delta H_{1}$ and $\Delta H_{2}$ values of the samples with ferrous lactate were $0.891 \mathrm{~J} \mathrm{~g}^{-1}$ and $0.256 \mathrm{~J} \mathrm{~g}^{-1}$, exhibiting respective increments of $2.65 \%$ and $36.17 \%$ compared to those of the control. The reason for the lower $\Delta H_{1}$ value of the sample with the ions exposed to magnetic field may be that the binding of ferrous ions to proteins resulted in the denaturation of proteins in surimi. The value of $\Delta H$ and the transition temperature were then reduced due to protein denaturation. The formation of large ice crystal particles during freezing led to damage, protein denaturation, and structural

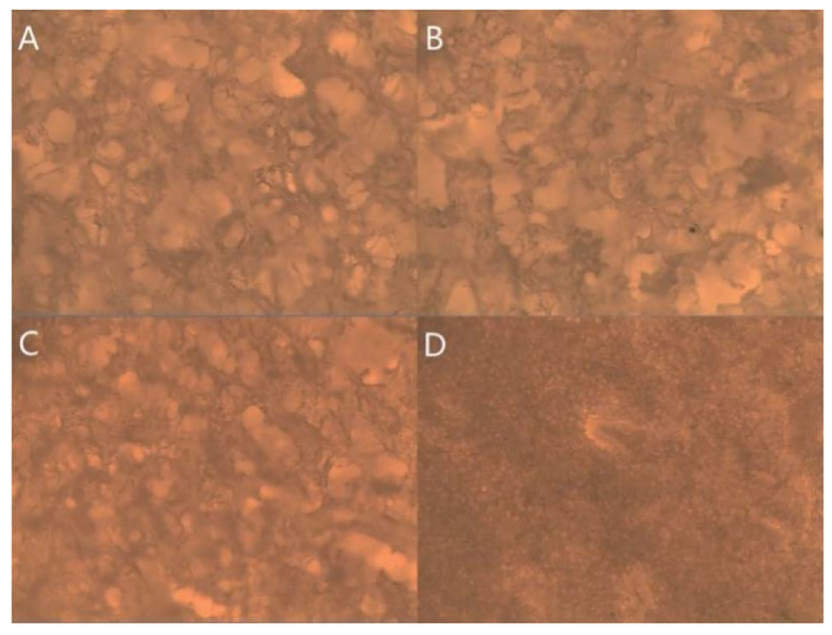

Fig. 5 Morphologies of the surimi after different treatments observed via optical microscope. (A) Control sample; (B) freeze-thawing under uniform magnetic field; (C) fresh sample; (D) freeze-thawing under uniform magnetic field plus ferrous ions. changes. However, the high thermal protein stability in surimi after oscillating uniform magnetic field-assisted freeze-thawing was associated with the formation of small ice crystals.

\subsection{Microstructure}

Fig. 5 presents the morphologies of the surimi samples after different treatments. After uniform magnetic field-assisted freeze-thawing, the sample with ferrous ions had the smallest pore size and the most uniform distribution. After the surimi was freeze-dried, the ice crystals sublimated, leaving pores in the original positions. Therefore, the pores in the tissue reflect the sizes of the ice crystal particles formed during freezing, and the distribution of ice crystals is an important index of frozen surimi product. The formation of large ice crystals can induce mechanical damage to muscle fibers, and thus an outflow of muscle exudates appeared. Consequently, the interaction of cellular components (e.g., lipids and proteins) and enzymes leads to protein denaturation and lipid degradation. ${ }^{31}$ The decreased ice crystal size was due to the rupturing of hydrogen bonds under the oscillating magnetic field, which was caused by the magneto-induced current. ${ }^{28}$ This phenomenon was prominent when metal ions were added because the conductivity of the sample was increased. To this effect, water molecule clusters were broken, and the sizes of the crystal nuclei therefore failed to reach the minimum nuclear radius during freezing, inhibiting the formation of large ice crystal particles.

\section{Conclusions}

The uniform magnetic field improved the quality of the freezethawed surimi. Specifically, the water-holding capacity was enhanced and thawing loss was decreased after the addition of ferrous ions to the samples. The denaturation temperature, denaturation enthalpy, and protein stability increased with the application of the oscillating magnetic field, and the added ferrous ions enhanced the effect of the magnetic field on the freeze-thawing process. Morphological observation revealed that ferrous ions combined with the uniform magnetic field significantly influenced surimi structure during freeze-thawing, resulting in the smallest pores in the sample. These findings suggest that the iron supplement induced an eddy current or magneto-induced current under the oscillating magnetic field due to electromagnetic induction. Therefore, the application of an oscillating magnetic field with high uniformity during freeze-thawing is an alternative method for the preservation of surimi.

\section{Conflicts of interest}

There are no conflicts of interest.

\section{Acknowledgements}

The study was financially supported by the National Key Research and Development Program of China (Grant No. 2017YFD0400404), the Open Fund of Beijing Key Laboratory for Food Non-thermal 
Processing (NTKF2018001), the Agricultural Science and Technology Independent Innovation Funds of Jiangsu Province (SCX(19)3367), and the Opening Foundation of Jiangsu Key Laboratory of Advanced Food Manufacturing Equipment and Technology (No. FM-201905). Our grateful thanks are due to Haoqi Zhang of Zhuyuan Primary School, Pudong, Shanghai for assistance with the preparation of surimi in her summer vacation and to Ms Xuerong Zhu for valuable discussion.

\section{References}

1 X. Zhou, T. Chen, H. Lin, H. Chen, J. Liu, F. Lyu and Y. Ding, Food Hydrocolloids, 2019, 90, 82-89.

2 H. J. Alipour, M. Rezaei, B. Shabanpour and M. Tabarsa, Food Hydrocolloids, 2017, 74, 87-96.

3 T. Petcharat and S. Benjakul, Food Hydrocolloids, 2017, 77, 746-753.

4 S. S. Shitole, A. K. Balange and S. S. Gangan, Int. Aquat. Res., 2014, 6(1), 55-66.

5 S. Wu, Int. J. Biol. Macromol., 2016, 93, 1118-1120.

6 G. Wenhong, H. Yangping, Z. Xin-an and A. Margaret, Int. J. Biol. Macromol., 2019a, 135, 839-844.

7 L. Cheng, D. W. Sun, Z. Zhu and Z. Zhang, CRC Crit. Rev. Food Technol., 2017, 57(4), 13.

8 M. Sadot, S. Curet, O. Rouaud, A. L. Bail and M. Havet, Int. J. Refrig., 2017, 80, 66-76.

9 A. Olmo, R. Baena and R. Risco, Int. J. Refrig., 2008, 31(2), 262-269.

10 F. Saideh, H. Nasser, K. Ezat, K. Javad and D. Mohsen, Int. J. Refrig., 2019, 99, 30-36.

11 S. Zhao, Z. Yang, L. Zhang, N. Luo and X. Li, J. Food Eng., 2018, 217, 24-33.

12 L. Otero, M. Pérez-Mateos, A. C. Rodríguez and P. Sanz, J. Food Eng., 2017a, 200, 87-94.

13 C. James, B. Reitz and S. J. James, Food Bioprocess Technol., 2015, 8(3), 702-708.

14 F. Fernández-Martín, M. Pérez-Mateos, S. Dadashi, C. M. Gómez-Guillén and P. D. Sanz, Innovative Food Sci. Emerging Technol., 2017, 44, 131-138.

15 F. Fernández-Martín, M. Pérez-Mateos, S. Dadashi, C. M. Gómez-Guillén and P. D. Sanz, Innovative Food Sci. Emerging Technol., 2018, 49, 176-183.
16 J. Liu, C. Fang, Y. Luo, Y. Ding and S. Liu, Food Hydrocolloids, 2018, 89, 668-673.

17 A. E. Lysionek, M. B. Zubillaga, M. J. Salgueiro, R. A. Caro, M. Segal, N. Shafran, N. Shapira and J. R. Boccio, J. Nutr. Sci. Vitaminol., 2002, 48(4), 315-317.

18 AOAC, Official Methods of Analysis of the Association of Official Analytical Chemists, Association of Official Analytical Chemists, Arlington, VA, 17th edn, 2000.

19 P. Rajendra, H. N. Sujatha and D. Devendranath, Biological effects of power frequency magnetic fields: neurochemical and toxicological changes in developing chick embryos, Biomagn. Res. Technol., 2004, (1), 1-9.

$20 \mathrm{~A}$. Gani and S. Benjakul, Impact of virgin coconut oil nanoemulsion on properties of croaker surimi gel, Food Hydrocolloids, 2018, 82, 34-44.

21 G. Wenhong, H. Rui and Z. Xin-An, J. Food Eng., 2019b, 240, 1-8.

22 J. H. Mok, W. Choi, S. H. Park, S. H. Lee and S. Jun, Int. J. Refrig., 2015, 50, 137-145.

23 L. Otero, A. C. Rodríguez and P. D. Sanz, J. Food Eng., 2017b, 217, 34-42.

24 H. Mi, B. Zhao, C. Wang, S. Yi, Y. Xu and J. Li, J. Sci. Food Agric., 2017, 97(14), 4807-4814.

25 Q. Sun, F. Sun, X. Xia, H. Xu and B. Kong, Ultrason. Sonochem., 2019, 51, 281-291.

26 D. C. Kang, X. Q. Gao, Q. F. Ge, G. H. Zhou and W. G. Zhang, Ultrason. Sonochem., 2017, 38, 317-325.

27 A. C. Rodríguez, C. James and S. J. James, Food Bioprocess Technol., 2017, 10(9), 1-7.

28 N. Manuchehrabadi, Z. Gao, J. Zhang, H. L. Ring, Q. Shao and F. Liu, Sci. Transl. Med., 2017, 9(379), eaah4586.

29 Y. Ali Arfat and S. Benjakul, Food Biosci., 2013, 3, 1-9.

30 P. Kaushik, K. Dowling, S. McKnight, C. Barrow, B. Wang and B. Adhikari, Preparation, characterization and functional properties of flax seed protein isolate, Food Chem., 2016, 197, 212-220.

31 G. Jia, X. He, S. Nirasawa, E. Tatsumi, H. Liu and H. Liu, J. Food Eng., 2017, 204, 18-26. 